\section{A British \\ Ophthalmological Surveillance Unit Study on metastatic endogenous endophthalmitis}

\begin{abstract}
Purpose Endogenous endophthalmitis (EE) is a rare but serious ocular infection caused by the seeding of bacteria into the eye from a source elsewhere in the body. Studies suggest that EE accounts for 2 to $8 \%$ of all endophthalmitis.

Methods A prospective observational study was conducted using the British Ophthalmological Surveillance Unit reporting system. Questionnaires were sent to reporting Ophthalmologists in the UK to assess incidence, underlying aetiology, eye findings, management, and final outcomes in endogenous endophthalmitis over a 12-month period within the British Isles.

Results Sixty two cases reported with 48 initial questionnaires returned and 25 6-month follow-up questionnaires returned. The median age of patients affected was 57 years with youngest aged 2 years and oldest aged 85 years. Twenty three were male and 24 were female. The median visual acuity in the affected eye was $3 \log$ MAR (range -0.1 to 5 ). Blood cultures were taken in 36 patients, $58 \%$ of which were positive. Vitreous biopsy was taken in 35 patients, $23 \%$ of which were culture positive. The visual function as assessed by visual acuity had significantly improved at 6 months with a median acuity of $0.18 \log$ MAR $(P=0.003)$.

Conclusions The survey demonstrates the severe nature of endogenous endophthalmitis in patients with active infection or with risk factors for infection. Our study has demonstrated that at least half of the patients who were treated had significant vision improvement.

Eye (2018) 32, 743-748; doi:10.1038/eye.2017.284; published online 12 January 2018
\end{abstract}

S Maling ${ }^{1}$, C King $^{1}$ and N Davies ${ }^{2}$

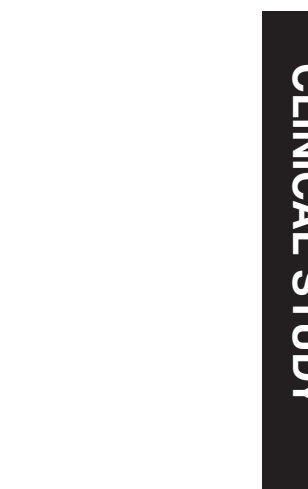

Introduction

Endogenous endophthalmitis (EE) is a rare but serious ocular infection caused by the seeding of bacteria into the eye from a source elsewhere in the body. Studies suggest that EE accounts for 2 to $8 \%$ of all endophthalmitis. ${ }^{1,2}$

A review of EE in 2003 showed that underlying medical conditions predisposed to ocular infection in 56 to $68 \%$ of cases including recent hospitalization, diabetes mellitus, urinary tract infection, immunosuppression (associated with underlying malignancy, neutropenia, and HIV (human immunodeficiency virus)), intravenous drug abuse (IVDA), and indwelling catheters. ${ }^{3,4}$ In another study in 2012, 90.9\% of patients reported a pre-existing condition of which diabetes mellitus was the most common $(50 \%) .^{5}$

EE can be caused by a bacteria or fungi. It has been shown in the literature that there is geographic variability as to the organisms causing EE. A study from USA reported fungi $(62 \%)$ to be more common than bacteria (38\%). ${ }^{6}$ Another study from Hong Kong, ${ }^{7}$ however, reported bacteria to be more commonly found as the predisposing pathogens compared to fungi (72.7 vs $27.3 \%$ ). The organisms identified also appear to have geographic variability with the suggestion that Gram-negative bacteria are the most common identified bacteria in East Asia accounting for $70 \%, 5$ in particular Klebsiella species. In North America and Europe the number of Gram-negative organisms identified has increased from $32 \%$ in 1986 to $52 \%$ in 2003. 8,9

The symptoms that are experienced and reported are also variable from mild discomfort and visual loss to severe pain and perception of light or worse vision. Misdiagnosis has been reported in 16 to $63 \%$ of cases with often
${ }^{1}$ Buckinghamshire Healthcare NHS Trust, Aylesbury, UK

${ }^{2}$ Chelsea and Westminster Hospital, London, UK

Correspondence:

S Maling, Ophthalmology

Department, Stoke

Mandeville Hospital,

Aylesbury, Buckinghamshire HP21 8AL, UK

Tel: +44 (0)1296 315000;

Fax: +44 (0)1296315037.

E-mail: sarahmaling@

me.com

Received: 4 October 2016 Accepted in revised form: 16 October 2017

Published online:

12 January 2018

Previous presentations: Royal College of Ophthalmologists Annual Congess Liverpool 2015. 
considerable delay to the commencement of appropriate treatment. ${ }^{3,9}$

Means of diagnosis vary and include blood cultures, aqueous samples, vitreous taps to full vitreous biopsies. Treatment also varies from intravenous antibiotic to topical management. It has been shown ${ }^{11}$ that systemic antibiotics achieve therapeutic levels in the eye, however, intravitreal antibiotics appear to be the mainstay of treatment. Vancomycin $(1 \mathrm{mg} / 0.1 \mathrm{ml})$ and Ceftazidime $(2.25 \mathrm{mg} / 0.1 \mathrm{ml})$ are the most commonly used. A review study showed that eyes that had a vitrectomy (PPV) are three times more likely to retain vision than those that did not. $^{3}$

EE cases have been reported as having poor visual outcome. It is reported that acuities of counting fingers or better was found in 22.2 to $41 \%$ of cases. ${ }^{10,11}$ Fifty five to $69 \%$ of cases in one study reported blindness, evisceration, or enucleation. ${ }^{3}$

Given the variability in causative agent, presentation, management, and outcomes of this rare condition we conducted a surveillance study to assess the nature, management, and visual outcomes of endogenous endophthalmitis in the British Isles.

\section{Materials and methods}

Data collection via the British Ophthalmological Surveillance Unit (BOSU) reporting unit (https://www. rcophth.ac.uk/standards-publications-research/thebritish-ophthalmological-surveillance-unit-bosu/). Reporting of cases was made via the BOSU return card system and the period of data collection was from December 2013 to December 2014. On receipt of a report, an initial questionnaire was posted followed by up to three reminder letters, if needed. After a period of 6 months a follow-up questionnaire was posted, again with up to 3 reminder letters if needed.

Data were collected on the following parameters: demographics; source of referral; presenting symptoms and signs (function and anatomy); investigations performed (both ocular and systemic); and diagnosis. Underlying systemic diagnoses were also reported. The treatment given both ocular and systemic was also specified.

For the follow-up questionnaire, the ocular status was reviewed and data were collected on the final visual outcome and ocular anatomical outcome.

Visual acuity converted into logMAR when given as a Snellen acuity. Following Holladay ${ }^{12}$ acuities of CF, HM, PL were included as $\log$ MAR 3, 4, and 5 , respectively.

\section{Results}

\section{Demographics}

Sixty two cases were reported and of these 48 initial questionnaires (77\%), and 25 6-month follow-up questionnaires (52\% of initial completed questionnaires) were returned. One case was reported in duplicate and excluded. The median age of the group was 56.9 years, with interquartile range 39.4-68.4 years. One patients' age was not reported, one patient was $<5$ years old, 2 patients aged between 6 and 18 years, 5 patients between 19 and 35 years, 16 patients between 35 and 60 years, and 22 patients older than 60 years. Twenty three were male and 24 were female. Ethnicity data showed 31 white British, 3 white other, 1 afro Caribbean, 3 Asian and the remainder undeclared. ASA grade was collected from the initial questionnaire. The median ASA grade of the patients whose second questionnaire was returned was 3, while the median ASA grade of those whose second questionnaire was not returned was 2. A Mann-Whitney $U$-test showed no statistical difference between the ASA grades of patients with returned second questionnaires in comparison with those without $(P=0.67)$.

\section{Referral}

The most common reason for referral was loss of vision (28) and also red eye (24), painful eye (13), positive blood culture only (3). All three of these patients were graded as ASA grade 4; one had a genitourinary infection and candidaemia, one had Guillan-Barre syndrome and Streptococcus group A, and the third had beta haemolytic Streptococcus from a septic arthritis of the wrist. The duration of symptoms was given as $<7$ days in 30 referrals, 2-4 weeks in 10 and $>4$ weeks in one referral.

\section{Initial clinical state}

The condition was unilateral in 42 cases ( 24 right eyes and 18 left eyes) and bilateral in 6 cases. Visual acuity was not assessable in nine 9 and the presenting visual acuity was reported in 32 questionnaires with median of $3 \log$ MAR units (range -0.079 to 5 ), 21/32 worse than 1 logMAR and $8 / 32$ better than $0.3 \log$ MAR. For patients with bilateral disease, the acuities were reported as 'unable to assess' in 2 of the 6 cases, bilaterally poor in two cases (logMAR 3 in both eyes of one case, $\log$ MAR 4 and 1.77 in another case), unilaterally poor in one case (logMAR 3 and -0.08$)$ and bilaterally good in one case (logMAR 0.18 and -0.08).

Thirty one cases reported intraocular pressure measurement. IOP was low $(<9 \mathrm{~mm} \mathrm{Hg})$ in $4 / 31$, normal (10-21 $\mathrm{mm} \mathrm{Hg})$ in $23 / 31$, high $(21-30 \mathrm{~mm} \mathrm{Hg})$ in $1 / 31$ 
Table 1 Presenting ocular signs

\begin{tabular}{llll}
\hline Parameter & Numbers & & \\
\hline Cellulitis & Preseptal: 2 & Orbital: 3 & \\
RAPD & no RAPD: 41 & RAPD: 6 & \\
Cornea & Clear:30 & Cloudy: 12 & Hypopyon: 14 \\
Anterior chamber & Clear: 4 & Cells: 35 & \\
Lens & Clear: 29 & Cataract: 8 & \\
Vitreous & Clear: 1 & Vitritis: 37 & Fibrin: 10 \\
Fundus & No view: 19 & & Abscess: 4 \\
Retina & Retinitis: 19 & Roth's spots: 3 & Pale: 0 \\
Choroid & Choroiditis: 8 & Swollen: 3 & \\
Optic nerve & Normal: 14 & & \\
\hline
\end{tabular}

Table 2 Systemic diagnosis and organisms

\begin{tabular}{|c|c|c|}
\hline Systemic condition & Organism & Laterality \\
\hline Diabetes and pharyngitis & S. aureus & \\
\hline Diabetes, septic arthritis and central line infection & S. aureus & \\
\hline Diabetes and IV drug use & None found & \\
\hline Diabetes and Ca Colon with colostomy & None found & \\
\hline Diabetes, renal failure and dialysis & None found & \\
\hline Diabetes and renal failure & S. aureus & \\
\hline $\begin{array}{l}\text { Diabetes, cardiac valve, renal failure and central line } \\
\text { infection }\end{array}$ & S. aureus & Bilateral \\
\hline GU infection & Neisseria meningitides & \\
\hline GU infection & Candida albicans & \\
\hline IV Drug user and scalp abscess & None found & Bilateral \\
\hline IV Drug user and endocarditis & Group A Streptococcus & \\
\hline IV drug user & None found & \\
\hline Cardiac valve endocarditis & S. aureus & Bilateral \\
\hline Cardiac valve endocarditis & Group B Streptococcus & \\
\hline Tooth abscess & None found & \\
\hline Septic arthritis (shoulder) & None found & \\
\hline Septic arthritis (wrist) & S. aureus & \\
\hline Septic arthritis (wrist) & $\begin{array}{l}\text { Beta haemolytic Streptococcus and coagulase-negative } \\
\text { Staphylococcus }\end{array}$ & \\
\hline Endocarditis & S. aureus & \\
\hline SLE, nephrotomy, acute renal injury & Candida albicans & \\
\hline Necrotic sacral sore & Aspergillus & \\
\hline Fungal foot infection & None found & \\
\hline Hip replacement surgery & None found & \\
\hline Cerebral abscess and infected carotid stent & Escherichia coli & \\
\hline Neck abscess & S. aureus & \\
\hline Ulcerative colitis and infected packed ileal pouch & Candida albicans & \\
\hline Stage 4 ovarian CA and chemotherapy & Group C Streptococcus & \\
\hline Meningitis & Pneuтососсиs & Bilateral \\
\hline Septicaemia & Pneumococcus & Bilateral \\
\hline Guillan-Barre and Sepsis & Group A Streptococcus & \\
\hline Cervical discitis & S. aureus and S. dysgalactiae & \\
\hline Pneumonia & Group A Streptococcus & \\
\hline Rheumatoid arthritis source unknown & None found & Bilateral \\
\hline Multiple sclerosis and URTI & S. epidermidis & \\
\hline Whipple's procedure and septicaemia & Rhodetorula & \\
\hline Unknown & None found & \\
\hline Unknown & None found & \\
\hline Unknown & S. milleri & \\
\hline Unknown & S. warheri & \\
\hline Unknown & Pneumococcus & \\
\hline Unknown & Candida, S. aureus, S. viridans, $H$. influenzae & \\
\hline
\end{tabular}


Table 3 Systemic antimicrobials

\begin{tabular}{lcc}
\hline Systemic antibiotics & Oral & Intravenous \\
\hline Flucloxacillin & & 6 \\
Gentamicin & & 3 \\
Ceftriaxone & & 5 \\
Ceftazidime & 1 & 1 \\
Cefotaxime & & 2 \\
Tazocin & & 5 \\
Vancomycin & & 1 \\
Meripenem & & 3 \\
Clindamycin & & 2 \\
Benzylpenicillin & 2 & 1 \\
Linezolid & 2 & \\
Ciprofloxacin & 1 & \\
Moxifloxacin & 2 & \\
Metronidazole & 5 & \\
Augmentin & & \\
Fluconazole & 5 & 11 \\
Ketonconazole & 1 & \\
Voriconazole & & \\
Other & & \\
\hline
\end{tabular}

and very high (>30 $\mathrm{mm} \mathrm{Hg}$ ) in 2/31 cases. The remaining clinical signs reported are shown in Table 1.

The underlying systemic diagnosis was wide ranging and presented in Table 2 with the causative organism where identified.

Blood cultures were taken in 36 patients, $58 \%$ of which were positive. An AC tap was taken in 17 patients, $12 \%$ of which were positive. A vitreous biopsy was taken in 35 patients, $23 \%$ of which were positive. Three-port vitrectomy was undertaken in 6 patients, 1 of which was positive. Thirty cases presented with duration of symptoms less than 7 days (4 bilateral cases) and in these a causative organism was found in 25 (83\%, 20 bacteria, 5 fungi). 11 cases presented with duration of symptoms 2-4 weeks ( 2 bilateral) and in these causative organisms were found in 4 cases ( $36 \%, 3$ bacteria, 1 fungus). Two cases presented with $>4$ weeks duration and no organisms were found. In 5 cases the duration of symptoms data were not completed and in these two reported bacterial organisms and in the remaining three cases none were found.

Antibiotic treatments were separated into systemic and ocular groups, with systemic subdivided into oral or intravenous treatments. The different antibiotics used are presented in Tables 3 and 4 .

Topical steroids were given in 31 cases and systemic steroid in 7 cases.

\section{Six-month results}

Of 48 initial questionnaires, a total of 25 second questionnaires were returned (52\%). If a return was not received a second and third questionnaire was sent. From
Table 4 Intravitreal antimicrobials

\begin{tabular}{lc}
\hline Antibiotic & Number \\
\hline Vancomycin and Amikacin & 7 \\
Vancomycin and Ceftazidime & 18 \\
Vancomycin & 1 \\
Ceftrixone & 1 \\
Amphotericin & 9 \\
Ganciclovir & 1 \\
Foscarnet & 1 \\
Other & 1 \\
None given & 16 \\
\hline
\end{tabular}

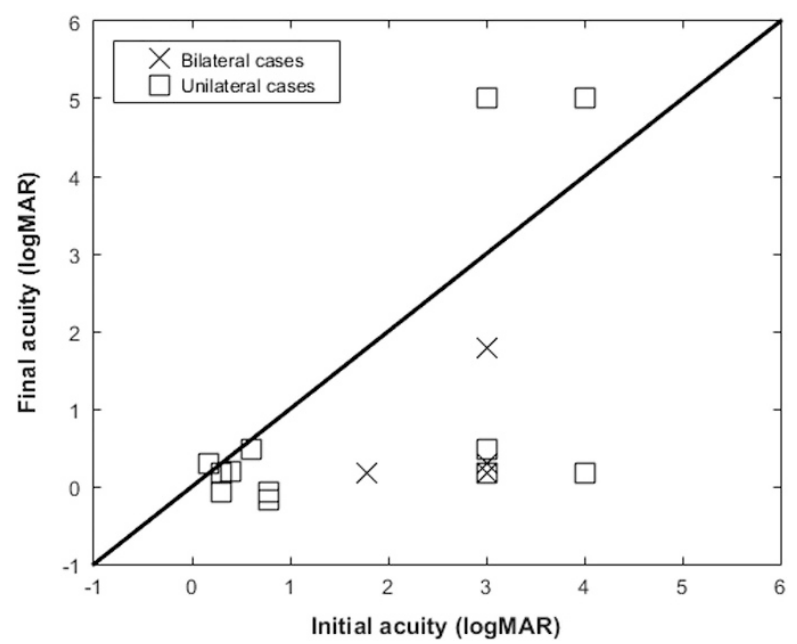

Figure 1 Initial and final reported visual acuities.

the returns received, during the 6-month time period, one patient had died, one patient had been registered as severely sight impaired, and no patients registered as sight impaired.

Acuity data were returned for 23 patients and median visual acuity at 6 months was $0.18 \log$ MAR (range -0.08 to $5 \log$ MAR). Four of 23 had $\log$ MAR acuity worse than 1.0 and 19/23 had acuity better than $0.3 \log$ MAR. In comparison with the initial acuity a paired $t$-test shows statistical significance with $P=0.003$ (Figure 1).

In the 4 patients with bad visual outcome two had afferent pupil defect (one eye NPL, other PL vision), one eye was phthisical. Three eyes were reported as having a pale optic nerve. The initial presentation was $<1$ week in two of these patients and 2-4 weeks in the other two. In three patients organisms were identified (Group A Streptococcus, Streptococcus pneumoniae, and Staphylococcus aureus) and three were treated with intravitreal and intravenous antibiotics. Two were intravenous drug users. The patient with $S$. aureus had a septic arthritis of the wrist and pre-existing advanced glaucoma. No vitreous biopsy or intravitreal treatment was given to this patient. For the remaining patients with a visual outcome 
better than $1.0 \log \mathrm{MAR}$, the ocular findings at 6 months showed two patients with ongoing anterior uveitis, one with cataract and eight had a hazy vitreous cavity. The ocular media were clear in the remainder of patients. The retina was reported as normal in 18 patients.

\section{Discussion}

Given a UK population of 64.1 million in 2014 and 62 reported cases for the reporting duration of 1 year, the estimated incidence of endogenous endopthalmitis is at least 1 per million per year. The results above show the data collected for 48 patients at initial presentation and 25 patients at the 6-month time point.

The presenting findings were typical of endogenous endophthalmitis with $94 \%$ of eyes showing reduced vision, involvement of the anterior chamber, and vitreous cavity. The reduced vision and other clinical signs would suggest that screening of high-risk patients is not necessary, if referral to ophthalmologists is made promptly if an ophthalmic problem becomes apparent.

The presenting level of visual acuity was very poor, typically counting fingers or hand movement. Some eyes however had a good measurable visual acuity. The majority of eyes had a vitreous biopsy taken and 23\% of these were positive for organism culture and this organism agreed with the underlying systemic infection where known.

A very wide range of systemic diseases and infectious processes were present in the presenting group (Table 2). All patients were receiving antibiotics either orally or intravenously and had the addition of intravitreal antibiotics following the sampling procedure. The most common combination was that of vancomycin and ceftazidime in 18 patients and vancomycin and amikacin in 7 patients.

The visual function as assessed by visual acuity had significantly improved over the group with the median visual acuity improving from $3 \log$ MAR to $0.08 \log$ MAR $(P=0.003)$. There were no differences in the eyes that improved in comparison with those that did not and we note that the visual acuity improved in 19 of the 25 patients with second questionnaires.

Four patients had bad visual outcomes with acuity measured worse than $1.0 \log \mathrm{MAR}$; these patients had infections with streptococcal species or Staphylococcus aureus and two were intravenous drug users. Three of these patients had intravitreal and intravenous antibiotics given. The patient with Staphylococcus aureus infection was reported as having pre-existing advanced glaucoma and no vitreous biopsy was performed nor intravitreal antibiotics given. This decision may have been made if the eye had very poor vision initially and therefore a poor (visual) prognosis.
Previous studies of endogenous endophthalmitis have shown final acuities of counting fingers or better in $22.2 \%$ to $41 \%$ of cases. ${ }^{10,11}$ Fifty to $69 \%$ of cases in one study reported blindness, evisceration, or enucleation. ${ }^{3}$ In our study, the final reported visual outcomes showed improvement in vision in 18 out of 25 cases where both initial and final acuities were reported. These data suggest that visual improvement is possible with treatment. Final acuity data however were not available for 23 cases as questionnaires were not returned despite the reminders being sent and this is a weakness of the study.

It is probable that the visual outcomes from the 23 patients whose 6-month acuity data were not available were poorer than in those for whom data were available. Although the ASA grades were similar in the patients with returned and non-returned second questionnaires, this could be because of higher morbidity and mortality in the non-returned group as well as a potential reluctance on the part of respondents to return the questionnaire on patients for whom the outcome was poor.

Data from the second questionnaires show one patient had died, giving a mortality rate in the first 6 months of $4 \%$. The mortality rate in another study was $5 \%,{ }^{3}$ indicating that most patients survive the acute episode.

In conclusion, we recommend that the standard of care should be that all patients referred from physicians with suspected endogenous endophthalmitis are seen promptly and treated aggressively with vitreous biopsy and intravitreal antibiotics to give the best opportunity for the infection to be eradicated and ocular damage limited, while continuing appropriate systemic antibiotics for the underlying infection. Treatment may give an opportunity for visual recovery, but significant morbidity and mortality remain serious concerns in patients with this condition. ${ }^{13}$

\section{Summary}

What was known before

- Endogenous endophthalmitis is rare associations with medical pathologies vary widely in the literature. Reported outcomes are variable.

What this study adds

- Endogenous endophthalmitis is associated with a range of medical pathologies. Visual outcomes are poor. This highlights the need for prompt diagnosis and treatment in suspected cases.

\section{Conflict of interest}

The authors declare no conflict of interest. 


\section{References}

1 Chee SP, Jap A. Endogenous endophthalmitis. Curr Opin Ophthalmol 2001; 12(6): 464-470.

2 Rachitskaya AV, Flynn HW, Davis JL. Endogenous endophthalmitis caused by salmonella serotype B in an immunocompetent 12-year-old child. Arch Ophthalmol 2012; 130(6): 802-804.

3 Jackson TL, Eykyn SJ, Graham EM, Stanford MR. Endogenous bacterial endophthalmitis: A 17-year prospective series and review of 267 reported cases. Surv Ophthalmol 2003; 48(4): 403-423.

4 Sadiq MA, Hassan M, Agarwal A, Sarwar S, Toufeeq S, Soliman MK et al. Endogenous endophthalmitis: diagnosis, management, and prognosis. J Ophthalmic Inflamm Infect 2015; 5: 32.

5 Wu ZH, Chan RP, Luk FO, Liu DT, Chan CK, Lam DS et al. Review of clinical features, microbiological spectrum, and treatment outcomes of endogenous endophthalmitis over an 8-year period. J Ophthalmol 2012; 2012: 265078.

6 Schiedler V, Scott IU, Flynn Jr HW, Davis JL, Benz MS, Miller D. Culture-proven endogenous endophthalmitis: Clinical features and visual acuity outcomes. Am J Ophthalmol 2004; 137: 725-731.
7 Wong JS, Chan TK, Lee HM, Chee SP. Endogenous bacterial endophthalmitis: an east Asian experience and a reappraisal of a severe ocular affliction. Ophthalmology 2000; 107: 1483-1491.

8 Sridhar J, Flynn Jr HW, Kuriyan AE, Miller D, Albini T. Endogenous fungal endophthalmitis: Risk factors, clinical features, and treatment outcomes in mold and yeast infections. J Ophthalmic Inflamm Infect 2013; 3(1): 60.

9 Binder MI, Chua J, Kaiser PK, Procop GW, Isada CM. Endogenous endophthalmitis: An 18-year review of culturepositive cases at a tertiary care center. Medicine (Baltimore) 2003; 82(2): 97-105.

10 Riddell J 4th, Comer GM, Kauffman CA. Treatment of endogenous fungal endophthalmitis: Focus on new antifungal agents. Clin Infect Dis 2011; 52(5): 648-653.

11 Greenwald MJ, Wohl LG, Sell CH. Metastatic bacterial endophthalmitis: a contemporary reappraisal. Surv Ophthalmol 1986; 31(2): 81-101.

12 Holladay JT. Proper method for calculating average visual acuity. J Refract Surg 1997; 13(4): 388-391.

13 Lingappan A, Wykoff CC, Albini TA, Miller D, Pathengay A, Davis JL et al. Endogenous fungal endophthalmitis: Causative organisms, management strategies, and visual acuity outcomes. Am J Ophthalmol 2012; 153: 162-166.e1. 